\title{
Indicadores de evaluación presupuestaria. Caso de estudio unidad municipal comunales Cienfuegos
}

\section{Budget evaluation indicators. Communal municipal unit case study Cienfuegos}

\author{
MSc. Milagros de la Caridad Mata Varela ${ }^{1}$ \\ mmatavarela@gmail.com \\ MSc. Johana Meza Salvatierra ${ }^{1}$ \\ ing johanna_meza@hotmail.com \\ MSc. Odalys del Carmen Toledo Rodríguez \\ odaca1964@gmail.com
}

Recibido: 1/12/2017, Aceptado: 1/02/2018

\begin{abstract}
RESUMEN
La investigación tiene como objetivo evaluar el proceso presupuestario a partir de un conjunto de indicadores y un índice integral. Se presenta el estado del arte relacionado con la conceptualización del proceso presupuestario, así como sus principales regularidades y particularidades, unido al empleo de ocho indicadores y un índice integral que posibilitan el análisis, seguimiento y evaluación del proceso, soportados en una estructura metodológica desarrollada y debidamente validada, posteriormente se demuestra su viabilidad en la Unidad Presupuestada Municipal Comunales Cienfuegos. Se utilizaron métodos teóricos y empíricos; unido a la revisión documental y el empleo de herramientas de agregación para las valuaciones y matemáticos como el método de valores límites. Todos permitieron concluir sobre la existencia de servicios generadores de ingresos, que posibilitan la redistribución del presupuesto notificado, unido a la evaluación de mínima integralidad del proceso en la unidad objeto de estudio.
\end{abstract}

Palabras clave: evaluación, integralidad, presupuesto, proceso

\section{ABSTRACT}

The objective of this research is to evaluate the budget process based on a set of indicators and a comprehensive index. It presents the state of the art related to the conceptualization of the budget process, as well as, its main regularities and particularities, together with the use of eight indicators and an integral index that enables

\footnotetext{
${ }^{1}$ Docente de la Universidad Metropolitana del Ecuador
}

Revista científica Ciencia y Tecnología Vol 18 No 18 págs. 103-113 http://cienciaytecnologia.uteg.edu.ec 


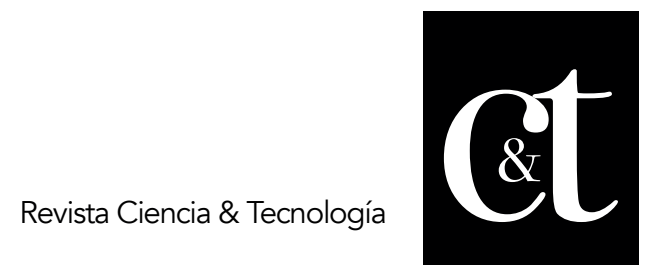

No. 18, 30 de abril de 2018

ISSN impreso: 1390 - 6321

the analysis, monitoring and evaluation of the process, supported by a developed and validated methodological structure, later its viability is demonstrated in the Municipal Budget Unit Cienfuegos. Theoretical and empirical methods were used; together with the documentary review and the use of aggregation tools for valuations and mathematics as the limit value method. All of them allowed us to conclude on the existence of income generating services, which make possible the redistribution of the notified budget, together with the evaluation of minimum integrality of the process in the unit under study.

Keywords: evaluation, integrality, budget, process

\section{Introducción}

Según Arellano (2006), la política fiscal es la más política de las políticas económicas, involucra la capacidad de disciplinarse ante necesidades múltiples y numerosos beneficiarios y grupos de interés cuando se cuenta con recursos limitados, es el mejor exponente de la capacidad de gobernabilidad de un sistema económico social, además de ser un instrumento de política macroeconómica. La necesidad de orientar el funcionamiento del sector público hacia la obtención eficiente de resultados para Granados, Larraín y Rodríguez (2013), implica revisar las prácticas en un sinnúmero de países, tanto en desarrollo como desarrollados. Estas condiciones demandan mayor accionar del gobierno, donde las tradicionales tareas de planificación, presupuesto y evaluación cobran una nueva dimensión, dejando de ser labores meramente administrativas, internas del gobierno, para transformarse en actividades estratégicas, y que reflejan la pertinencia y eficiencia de las políticas públicas que inciden en el bienestar social.

Así, las diferentes fases que conforman el proceso presupuestario tienen que ser capaces de retroalimentarse entre sí, conformando un todo coherente. Para completar el proceso, se requiere que exista además evaluación periódica, tanto para monitorear la ejecución presupuestaria y verificar si la misma refleja adecuadamente lo planificado, como para determinar si es pertinente revisar lo planificado. En Cuba el funcionamiento efectivo del gobierno en sus distintos niveles constituye un elemento importante para garantizar su perfeccionamiento económico, aspecto que se refuerza con la implementación de los lineamientos de la política económica y social del Partido y la Revolución, especialmente en lo relacionado con la disminución del gasto público y de las unidades presupuestadas hasta el número mínimo que garantice el cumplimiento de las funciones asignadas, con prevalencia de criterios de máximo ahorro del Presupuesto del Estado para garantizar un servicio eficiente y de calidad: lineamientos 256, 257 y 258 (PCC, 2016).

Dentro de las disimiles funciones del estado están las referidas a la limpieza y ornato público, tratamiento y disposición final de los residuos sólidos urbanos (RSU), entre otros. Especialmente los RSU constituyen un problema ambiental y de salud a nivel internacional, que imponen a los gobiernos atención diferenciada. Estas razones unido a los esfuerzos de los gobiernos locales para mantener las asignaciones presupuestarias independientemente del nivel de subordinación, justifica evaluar el desempeño del presupuesto en la Unidad Municipal Comunales Cienfuegos. En este contexto, el presente trabajo trata de dar respuesta al siguiente cuestionamiento ¿Cómo evaluar integralmente el proceso presupuestario en las unidades del sector público?, igualmente el objetivo está dirigido a evaluar integralmente el proceso presupuestario a partir de un conjunto de indicadores y un índice integral en las unidades del sector público. 


\section{Desarrollo}

Se realizó una investigación no experimental, durante los años 2013 al 2015. Se tuvo en consideración referentes teóricos internacionales y nacionales relacionados con esta temática, los documentos normativos para la actividad de las unidades presupuestadas, las metodologías que establecen los contenidos de las fases del proceso presupuestario, las resoluciones, además de las clases instructivas emitidas por el Ministerio de Finanzas y Precios de Cuba. Como parte del diagnóstico realizado fueron revisados los informes correspondientes a acciones de control realizadas a la liquidación del presupuesto durante los años 2013, 2014 y 2015 en la Dirección Municipal de Comunales Cienfuegos.

Para evaluar integralmente la actividad presupuestaria, a partir de indicadores por fase, se consideró seleccionar la metodología de Furniel (2015) por adaptarse a los requerimientos de la investigación, criterio que se sustenta en: la metodología se puede aplicar en cualquier unidad presupuestada del país y provee un sistema de indicadores por fase con un enfoque integral del proceso, en busca de la mejora continua, que responde a los lineamientos de la política económica y social del Partido y la Revolución.

Se utilizan ocho indicadores, que están agrupados por las diferentes fases del proceso presupuestario, y un índice integral, sustentados en un procedimiento metodológico dado por tres momentos que permite su correcta aplicación. Estos momentos son: selección del grupo de trabajo, diagnóstico del proceso presupuestario y definición de los indicadores y el índice integral (Figura 1).

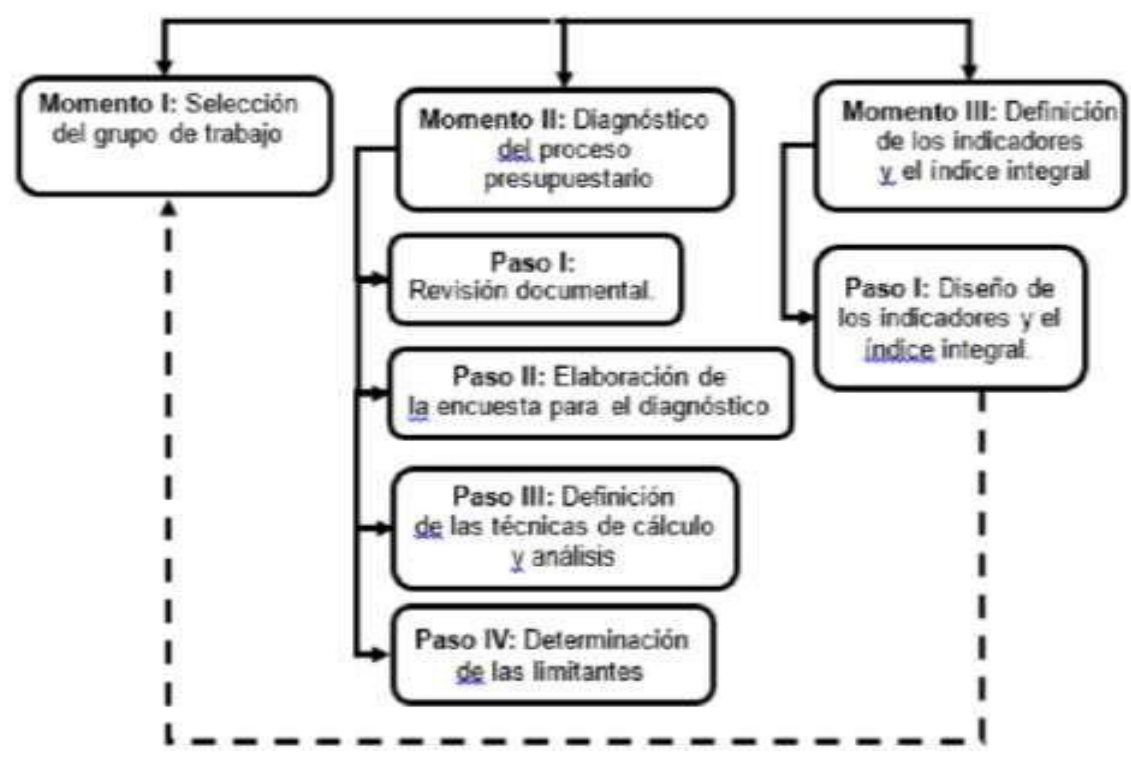

Figura 1. Metodología empleada en la elaboración del procedimiento Fuente: Tomado de Furniel (2015)

Los indicadores de evaluación por fases del proceso presupuestario y sus fórmulas de cálculo se relacionan en la Tabla 1. 
Tabla x: Sisteme ce indicadores por tases".

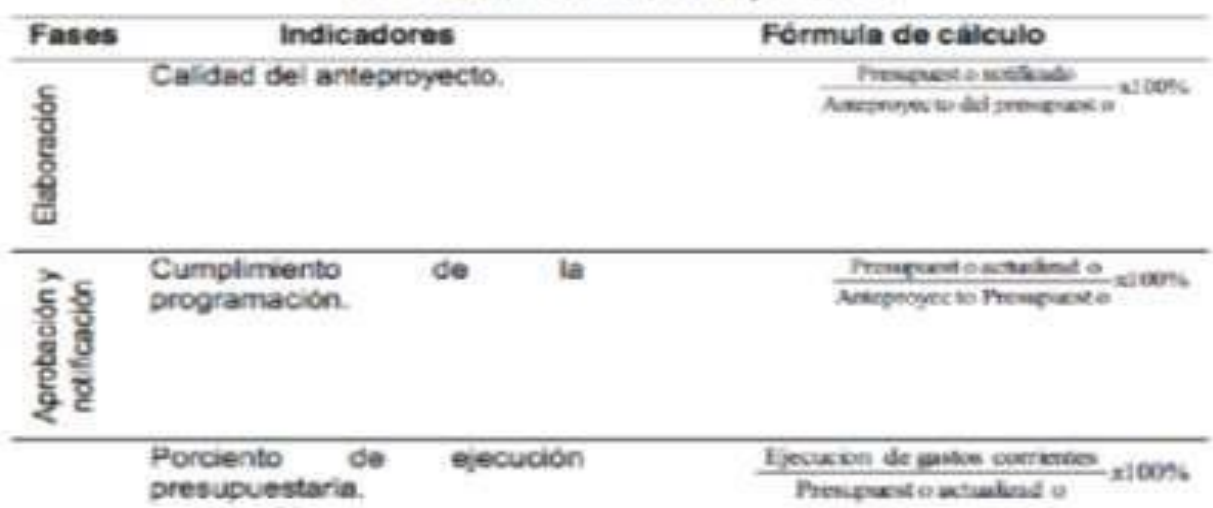

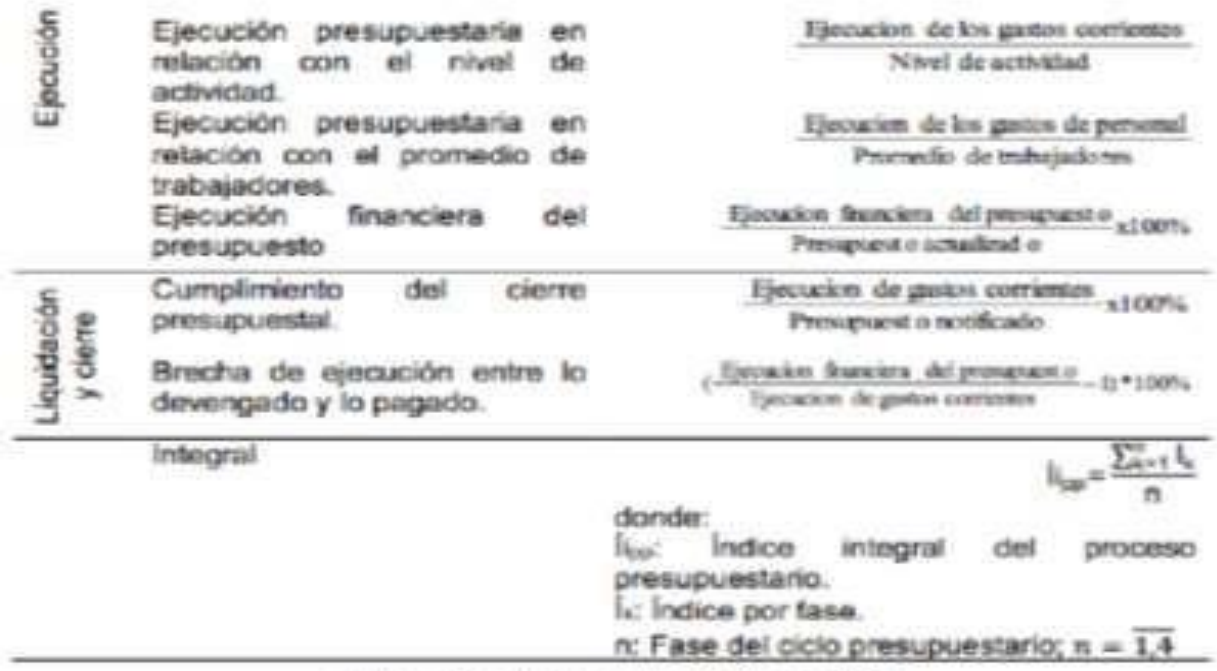

¿Elaboraclin propla a partir de Furminl (asis).

Luego es necesario estandarizar los indicadores y construir los índices por fases e integral. El procedimiento implica la realización de tres pasos: (a) Identificación de indicadores válidos y de la fuente de información correspondiente; (b) Determinación de los valores mínimos (Vi) y máximos (Vs), llamados genéricamente valores límites, para algunos de los indicadores, dígase: cumplimiento de la programación; ejecución presupuestaria \& promedio de trabajadores; cumplimiento del cierre presupuestal y brecha de ejecución devengado y pagado, a través de la expresión 1 ; y (c) Cálculo del índice integral, como resultado del promedio de los índices por fases. 


Revista Ciencia \& Tecnología

No. 18, 30 de abril de 2018

ISSN impreso: 1390 - 6321

$$
P=\frac{\left(V_{x}-V_{D}\right)}{\left(V_{x}-V_{D}\right)} ; \text { expresion } 1
$$

El resultado de este índice será $0 \leq$ Íipp $\leq 1$, lo que permite establecer rangos para evaluar el comportamiento del proceso presupuestario en relación a la integralidad. Para Medianero (2010) si el índice integral de cualquier proceso y estandarizado tiende al valor 1 , se califica de integral, si se acerca al valor cero entonces es no integral y si oscila alrededor del 0,5 entonces es considerado medianamente integral, por su parte las autoras Furniel (2015) y Mata (2016) consideran la incorporación de, dos nuevos valores en torno a las cifras 0,67 y 0,85 a través de los resultados del Íipp, los rangos se enuncian a continuación:

- No integral, para valores del Íipp estrictamente menor que 0,5;

- Mínima integralidad, para valores del Íipp en el intervalo $[0,5 ; 0,67[$;

- Casi integral, para valores del Íipp en el intervalo [0,67; 0,85[ e;

- Integral, para valores del Íipp mayor e igual que 0,85.

\section{Resultados}

En correspondencia con los contenidos abordados, se presentan los resultados para cada uno de los indicadores por fases y para el periodo de estudio. En la fase de elaboración el indicador Calidad del anteproyecto en los años 2013 y 2014 es calificada como buena, donde los resultados se mueven en el intervalo definido entre el $81 \%$ y el $99 \%$ de lo notificado, sin embargo, en el año 2015 la calidad del anteproyecto decrece con un valor relativo del $76 \%$ considerado como regular.

Para la fase de aprobación y notificación el presupuesto actualizado se encuentra por encima del presupuesto notificado, con una tendencia creciente valorada en 1324,40 MCUP, sin embargo, el presupuesto notificado muestra un decrecimiento de 49,9 MCUP. Las modificaciones estuvieron motivadas por la ocurrencia de eventos epidemiológicos, reparación y mantenimiento de viales de parques, micro parques, funeraria, cementerios, paradas y locales administrativos. Durante la fase de ejecución se dejan de ejecutar 247 MCUP y 153,7 MCUP en los años 2013 y 2014 respectivamente, con un $1 \%$ de inejecución a cuenta de la actividad de viales; como argumentos valorados se presenta la paralización de la actividad fundamental para trasladar la fuerza ejecutora a otros municipios; en el año 2015 la ejecución presupuestaria es al $100 \%$ por lo que se considera favorable para el periodo en estudio (Tabla 2). 
Tabla 2: Indicador 3. Porciento de ejecución presupuestarla".

\begin{tabular}{lrrr}
\hline Partidas e indicadores & 2013 & 2014 & 2015 \\
\hline Ejecución de Gastos Corrientes (MCUP) & 17966,7 & 17771,5 & 19430,1 \\
Presupuesto Actualizado (MCUP) & 18120,4 & 18018,5 & 19444,8 \\
Porciento de ejecución presupuestaria (\%) & 99 & 99 & 100 \\
\hline
\end{tabular}

"Elaborado a partir de los estados financieros y el presupuesto del actualizado.

El indicador porciento de ejecución presupuestario en relación con el nivel de actividad es del $100 \%$, sin embargo, el mismo se encuentra enmascarado por una deficiente normación del mismo, que estipula $0.75 \mathrm{~kg}$ de basura diaria por habitante y donde no se ha incluido la generación de RSU del sector cuenta propista y el turismo en el municipio Cienfuegos (Tabla 3).

Tabia 3: Indicador 4, Ejecución presupuestaria en reiación con el nivel de actividad".

\begin{tabular}{lrrr}
\hline Partidas e indicadores & 2013 & 2014 & 2015 \\
\hline Ejecución de Gastos Corrientes (MCUP) & 1043,8 & 549,9 & 496,4 \\
Nivel de actividad (MCUP) & 1070,7 & 550,0 & 496,4 \\
Areas Verde & 97 & 100 & 100 \\
Ejecución de Gastos Corrientes (MCUP) & 684,7 & 2123,7 & 2131,6 \\
Nivel de actividad (MCUP) & 684,7 & 2124,0 & 2160,0 \\
Residuos Sólidos Urbanos & 100 & 100 & 99 \\
Ejecución de Gastos Corrientes (MCUP) & 0,0 & 196,0 & 226,2 \\
Nivel de actividad (MCUP) & 0,0 & 196,0 & 266,3 \\
Reapile de Vertederos & $\ldots$ & 100 & 85 \\
Total de gastos (MCUP) & 1728,5 & 2869,6 & 2854,2 \\
Total Nivel de actividad (MCUP) & 1755,4 & 2870,0 & 2922,7 \\
Ejecución presupuestaria en relación con el & & & \\
nivel de actividad (\%) & 100 & 100 & 100 \\
\hline
\end{tabular}

"Elaborado a partir de los estados financieros y los niveies de activided.

Para el caso especial del indicador de ejecución presupuestaria en relación con el promedio de trabajadores se aprecia una disminución en el promedio de trabajadores y un aumento en el gasto de personal, con sobrevaloraciones de los subsidios por encima de lo planificado en 61600 CUP y el procesamiento de 616 certificados médicos (Tabla 4). 
ब बt

No. 18,30 de abril de 2018

ISSN impreso: 1390 - 6321

Tabla 4: Indicador 5. Ejecución presupuestaria en relación con el promedio de trabajadores".

\begin{tabular}{lrrr}
\hline Partidas e indicadores & 2013 & 2014 & 2015 \\
\hline Ejecución de los Gastos de Personal (MCUP) & 6218,4 & 6023,4 & 6296,9 \\
Promedio de Trabajadores & 1024 & 1018 & 1022 \\
$\begin{array}{l}\text { Ejecución presupuestaria en relación al promedio de } \\
\text { trabajadores (MCUP) }\end{array}$ & 6072,7 & 5920,0 & 6161,4 \\
\hline
\end{tabular}

"Elaborado a partir de los estados finaneieros y el promedio de trabajadores.

La ejecución financiera del presupuesto muestra un saldo positivo, crece de forma relativa de un $97 \%$ hasta $99 \%$. Se observan gastos por encima de lo notificado en los tres años de referencia (Tabla 5). El comportamiento sobrevalorado de la ejecución del gasto corriente respecto a la ejecución financiera de forma sostenida durante el periodo de estudio está condicionado por dejarse de financiar 378.1 MCUP quedando comprometidos para el próximo año (Tabla 6).

Tabla 5 : Indicador 7. Cumplimiento del cierre presupuestar".

\begin{tabular}{lrrr}
\hline Partidas e indicadores & 2013 & 2014 & 2015 \\
\hline Ejecución del Gasto Corriente (MCUP) & 17966,7 & 17771,5 & 19430,1 \\
Presupuesto Notificado (MCUP) & 17322,9 & 17273.0 & 16428,4 \\
Cumplimiento del cierre presupuestal (\%) & 104 & 103 & 119 \\
\hline
\end{tabular}

•Elaborado a partir de los estados financieros y el presupuesto notificado.

Tabia 6: Indicador 6. Brocha de ejecución entre el devengado y ei pagado*:

\begin{tabular}{lrrr}
\hline Partidas e indicadores & 2013 & 2014 & 2015 \\
\hline Ejecución Financiera del Presupuesto (MCUP) & 17588,6 & 17697,8 & 19285,1 \\
Ejecución de Gastos Corrientes (MCUP) & 17966,7 & 17771,5 & 19430,1 \\
Brecha de ejecución entre el devengado y el & -2.1 & -0.41 & -0.75 \\
pagado (\%) & & & \\
\hline
\end{tabular}

"Elaborado a partir de la ejecución financiera y los estados financieros.

Los resultados de los indicadores obtenidos a través de la evaluación realizada y estandarizada posteriormente muestran la mejora sustancial de todos los indicadores durante el año 2015, solo la calidad del anteproyecto y la brecha de ejecución de lo devengado y lo pagado disminuye respecto a los años anteriores. De igual manera y como resultado de la evaluación del presupuesto en la Unidad Municipal Comunales Cienfuegos (índices), se advierte que el índice de la fase de elaboración es el único que manifiesta una contracción en el último año respecto a los anteriores, con un crecimiento sostenido del índice integral durante el periodo de análisis (Figura 2). 


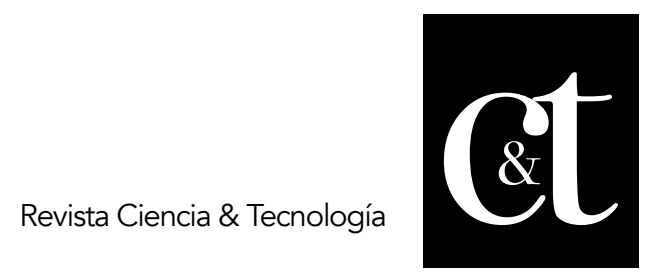

No. 18, 30 de abril de 2018

ISSN impreso: 1390 - 6321

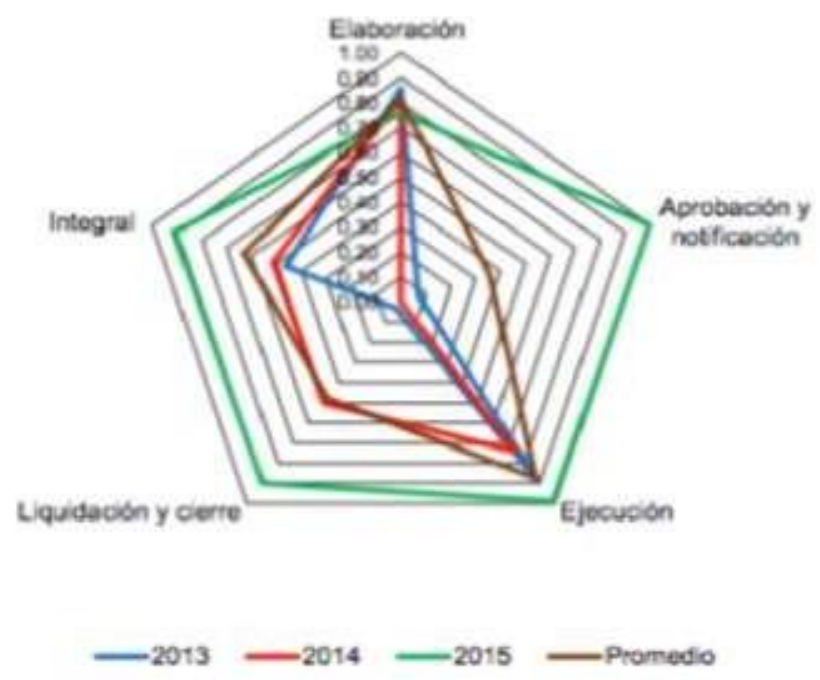

Figura 2. Índices por fases e integral

Por último, toda serie histórica asociada a una variable describe un comportamiento funcional que posibilita su proyección hacia el futuro, este postulado se aplica también a los índices por fases, que describen, además, comportamientos polinomiales de grado dos y lineales creciente y decrecientes según los casos; especialmente el índice integral experimenta una tendencia creciente, con una trayectoria polinómica de grado dos para el periodo en estudio (Figura 3).

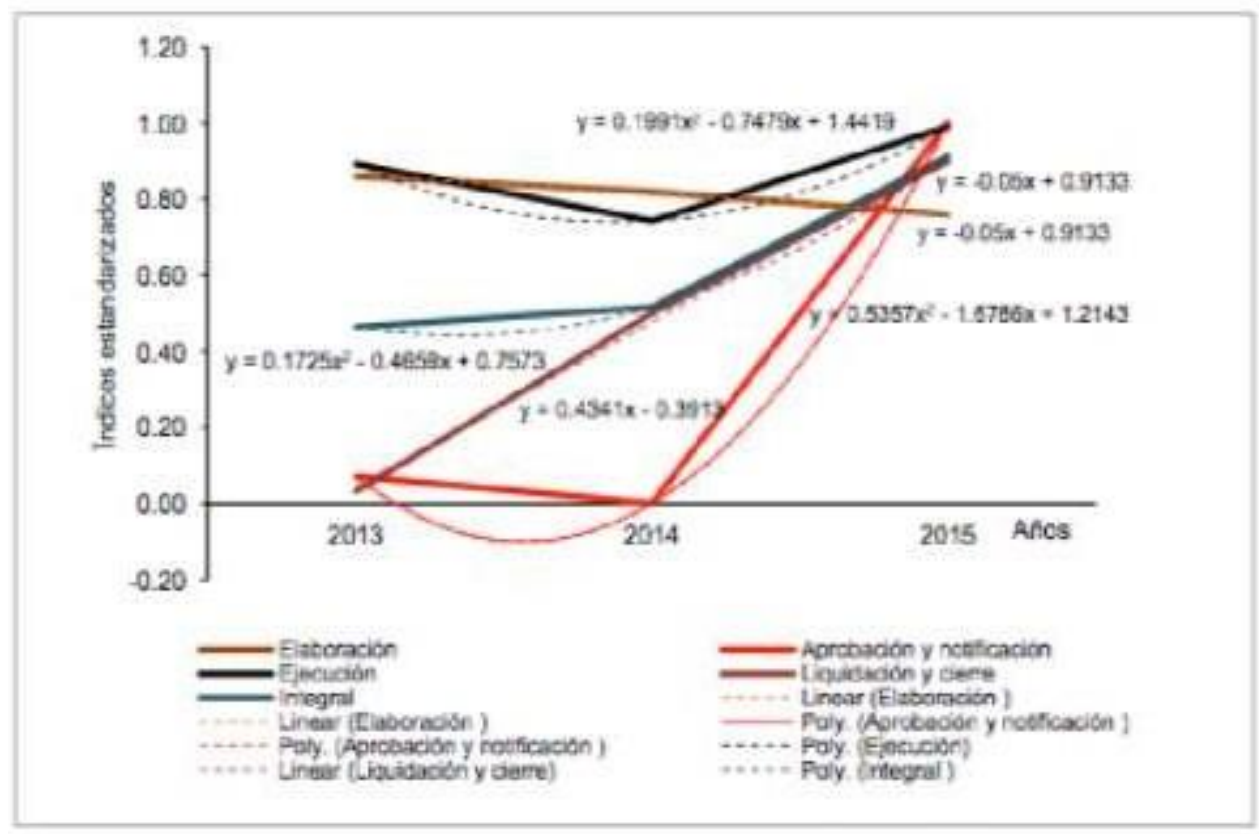

Figura $3^{*}$, Comportamiento tendencial para el indlce integral. Fuente: Elaborado a partir del procesamiento con el empleo del Microsoff Office Excel. 
बत

No. 18,30 de abril de 2018

ISSN impreso: 1390 - 6321

Por último, se emite el Informe valorativo que permite identificar causas y condiciones de las deficiencias detectadas entre las que se encuentran las siguientes:

- Incumplimiento de la Resolución 254/2013 del Ministerio de Finanzas y Precios.

- Deficiente defensa ante el organismo superior de las cifras ante proyectadas.

- Incorrecta planificación en la notificación del presupuesto.

- Carencia de análisis de los costos de los servicios que prestan.

- Falta de actualización de la norma de generación de residuos.

- Escasa visión de los lineamientos de la política económica y social del Partido y la Revolución.

Teniendo en cuenta los resultados obtenidos se evalúa de mínima integralidad el proceso presupuestario al alcanzar un indicador general de 0,63 , incidiendo en este resultado las fases de aprobación y notificación y de liquidación y cierre, las que alcanzan valores puntuales promedio de 0,36 y 0,48 respectivamente. La unidad, proyectó un presupuesto para los años 2013 al 2015 valorado en 62810 000,00 CUP, con una calidad por año de $86 \%, 82 \%$ y $72 \%$ respectivamente. En el período analizado se notifica un presupuesto de 51024 300,00 CUP, con un decrecimiento planificado con relación a años anteriores.

Los ingresos proyectados en el año 2014 no cumplen con las indicaciones del anteproyecto con un valor menor al del año anterior. El presupuesto actualizado para el periodo fue de 55583 700,00 CUP, y recibió modificaciones por valor de 4144 000,00 CUP, condicionado por la ejecución de actividades diversas y dirigidas fundamentalmente a reparaciones y mantenimientos constructivos, alquiler de equipos, reparación y mantenimiento de viales, campañas epidemiológicas (cólera y vectorial), así como acciones para contrarrestar los daños ocasionados por eventos meteorológicos con el 60 \% de las modificaciones efectuadas por este concepto; todos estos elementos impidieron el cumplimiento de los niveles de actividad en diferentes servicios programados.

Aun cuando el índice de calidad en la fase de planeación no refleja resultados alarmantes, se puede aseverar que existen problemas con la planificación presupuestaria, al aprobarse cifras por debajo de lo proyectado (11 785 700,00 CUP), sin lugar a dudas las afectaciones se reflejan en servicios sensibles como: limpieza de calles $(-13,1 \%)$, recogida de RSU $(-3,4 \%)$, ventas de plantas ornamentales $(-8,1$ $\%$ ) y la jardinería $(-33,8 \%)$, de ellos, los tres últimos generan ingresos promedios de 1689 709,00 CUP, concluyendo con una ejecución de 55168 300,00 CUP.

En el análisis realizado a los gastos de estos servicios se comprobó que la florería asume los gastos del Jardín Gigante que, a pesar de no tener producción planificada, incurre en gastos por conceptos diversos, dígase, compra de flores a terceros, fuerza de trabajo, electricidad, agua y otros. La producción durante el periodo de estudio fue de 285200 miles de decenas de flores de un plan promedio de 50600 y se comercializaron realmente un promedio de 535000 mil docenas de flores. Las áreas verdes del municipio Cienfuegos son 4723 miles de M2, y solo el $36 \%$ del área disponible es atendida por la unidad presupuestada de comunales. 
Por su parte la recogida de RSU a terceros es un servicio de recién institucionalización que ha experimentado una mejoría sustancial en cuanto a aseguramiento, número de clientes, y tarifas para el cobro del servicio que lo ubican en la tercera posición entre los más lucrativos dentro de la cartera. En el orden de la generación de utilidades se analizan los ingresos obtenidos con un total de 1689 709,00 CUP, con un crecimiento general.

Los servicios más lucrativos dentro de la cartera de negocio de comunales no tienen hoy tratamiento especial en la distribución del presupuesto del Estado, a pesar de ser capaces de cubrir con sus ingresos una parte importante de sus gastos, discrepando en este sentido con el lineamiento No.259 de la política económica y social del Partido y la Revolución.

\section{Conclusiones}

Luego del desarrollo de la investigación y como resultado de la misma se arribó a las siguientes conclusiones:

A partir de la puesta en práctica de los indicadores y de un índice integral, se evaluó de forma integral el proceso presupuestario en la Unidad Presupuestada Municipal Comunales Cienfuegos.

La implementación de los indicadores demuestra la existencia de servicios generadores de ingresos, que posibilitan una mejor redistribución del presupuesto notificado en la unidad objeto de estudio.

El proceso presupuestario en la Unidad Presupuestada Municipal Comunales Cienfuegos es evaluado de mínima integralidad con un valor general promedio de 0,63, incidiendo en este resultado las fases de aprobación y notificación y de liquidación y cierre.

La evaluación integral del proceso presupuestario, a partir de la aplicación de los indicadores y el índice integral realizada en la Unidad Presupuestada Municipal Comunales Cienfuegos, demuestra el cumplimiento de la hipótesis definida y de los objetivos planteados en la investigación.

\section{Referencias bibliográficas}

Arellano, J. P. (2006). Del déficit al superávit fiscal: razones para una transformación. CEP, Estudios Públicos.

Furniel, I. (2015). Indicadores para la evaluación integral del proceso presupuestario en las unidades presupuestadas cubanas (Tesis doctoral en Ciencias Contables y financieras). Universidad de Camagüey, Cuba.

Granados, S., Larraín, F., y Rodríguez, J. (2013). Planificación y presupuesto como herramientas de políticas pública. En Un mejor Estado para Chile (págs. 529 765). Santiago de Chile, Chile: Consorcio para la Reforma del Estado.

Mata, M. (2016). Administración financiera del ciclo de proyectos de inversión agropecuarios (Tesis doctoral en Ciencias Contables y financieras). Universidad de Camagüey, Cuba.

Medianero, D. (2010). Metodología de evaluación ex post. Pensamiento crítico, 13, 71- 90 . 


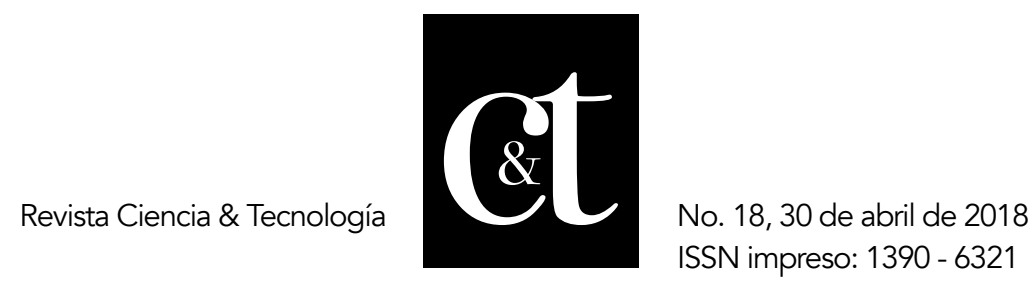

Partido Comunista de Cuba (2016). Actualización de los lineamientos de la Política Económica y Social. La Habana, Cuba: Oficina de publicaciones del Consejo de Estado.

Rodríguez, H. (2015). Indicadores de gestión presupuestarias para las universidades cubanas (Tesis presentada en opción al grado científico de Doctor en Ciencias Contables y Financieras). Universidad de Camagüey, Cuba.

Sánchez, L. (1997). Tratado de derecho financiero y tributario constitucional (Ediciones Jurídicas y Sociales, SA.). Madrid: Marcial Pons. 